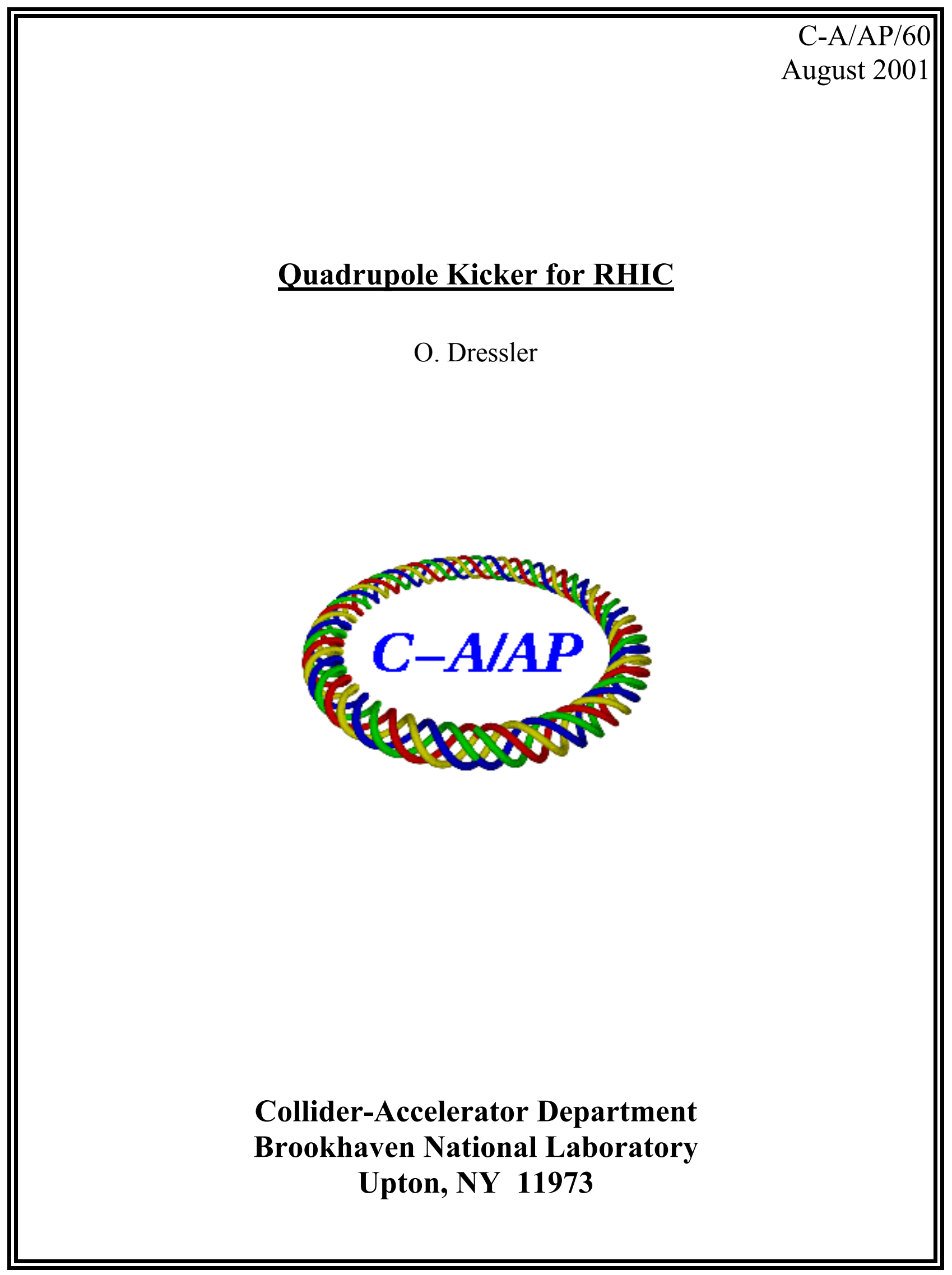




\section{Quadrupole Kicker for RHIC}

It is assumed that echo phenomena can be produced in RHIC with a quadrupole kicker exited a short time after an initial dipole kick. For that reason special air core quadrupole magnets were already installed in RHIC. The principal task was to design a pulse circuit what combines the electrical properties with the physical necessities.

In electrical terms of the kicker magnet is mainly described by applicable voltage and possession of inductivity. Both are limiting margins for the pulse circuit.

The inductivity of the magnet was measured with $105 \mu \mathrm{H}$. A feeding cable could have up to $20 \mu \mathrm{H}$. It was estimated that the applicable Voltage is $2000 \mathrm{~V}$. The period of revolution in RHIC requires a pulse length shorter than $20 \mu \mathrm{s}$. It was claimed to produce the highest available current with the pulse circuit (more than 50A). Because of the for this case very high inductance of the magnet the use of a sine half wave pulse circuit seemed to be the best solution.

\section{$\underline{\text { Circuit Diagram }}$}

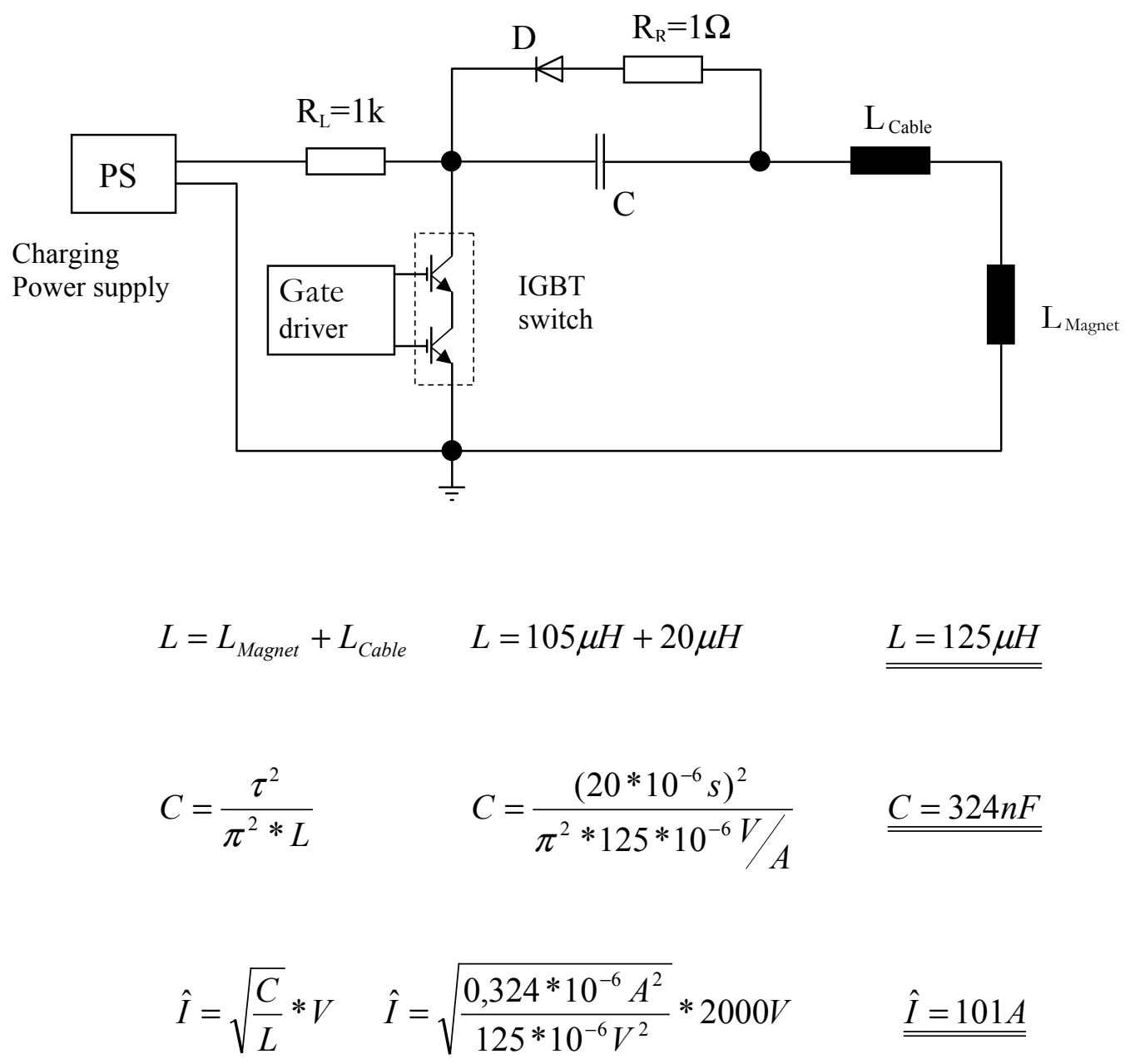


$\underline{\text { Block diagram }}$

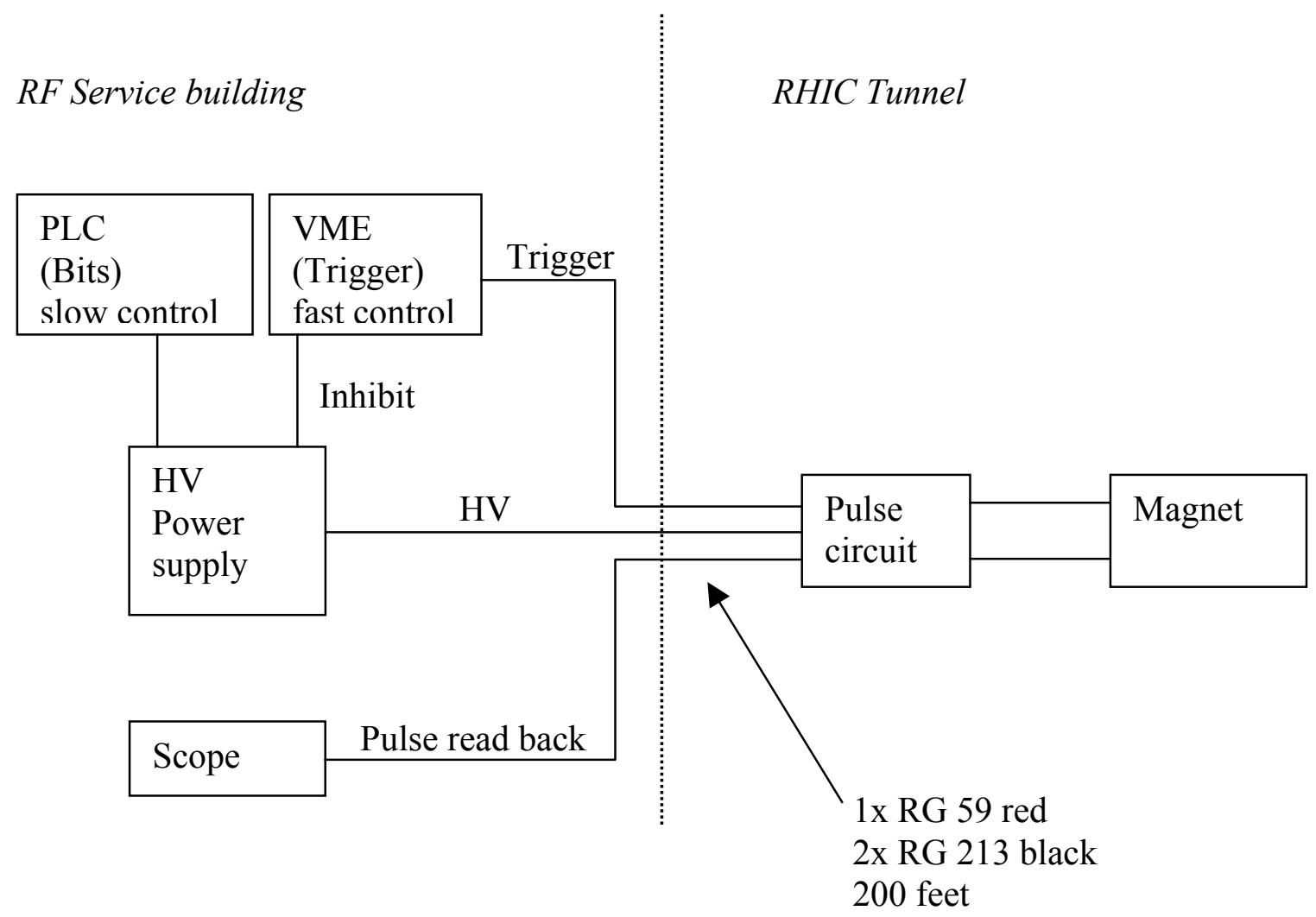

$\underline{\text { Gate driver circuit }}$

2 times to drive Dual IGBT Module CM100DU-34KA from Powerex, Inc.

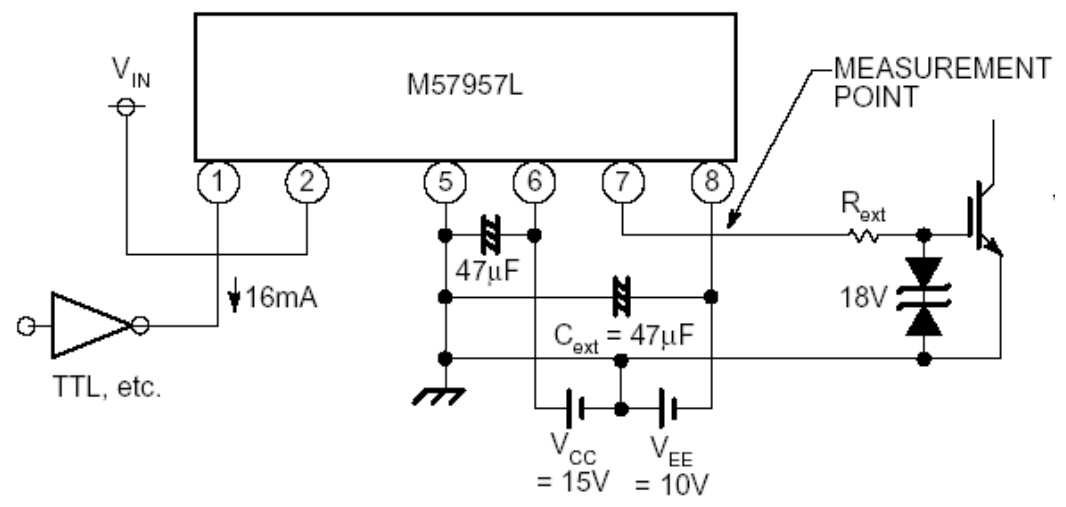




\section{$\underline{\text { Pulse shape }}$}

During our test we learned how to increase the pulse shape for the finally installation. The frequency of the resonant circuit is defined by $\mathrm{L}$ and $\mathrm{C}$. Since $\mathrm{L}$ is fix, only $\mathrm{C}$ can be changed change the frequency. The main part to influence the back swing performance is the reload circuit $R$. The choice of $R_{R}$ has to be made by evaluating the necessity for zero-crossing related to beam revolution, smooth approximation of the pulse current to the zero line without noise and how much reverse voltage the switch can bear.

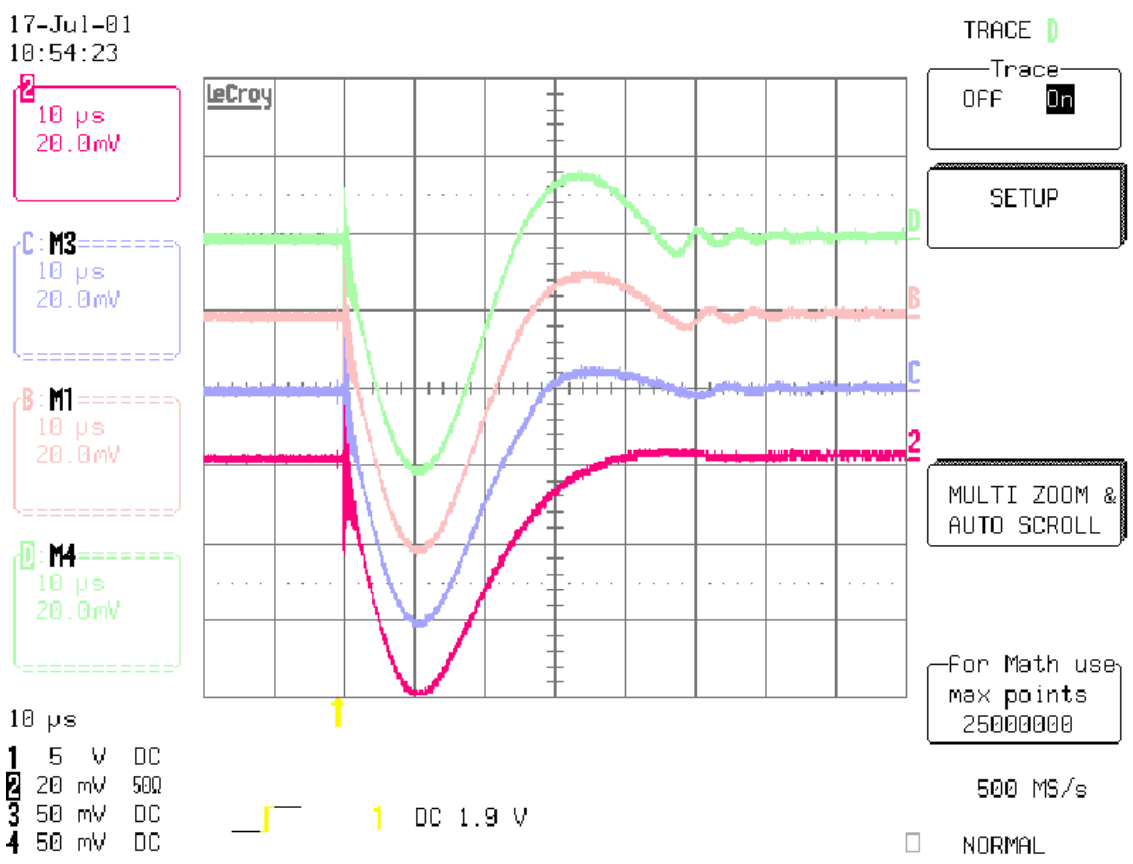

